\title{
Is it possible to predict late antepartum stillbirth by means of cerebroplacental ratio and maternal characteristics?
}

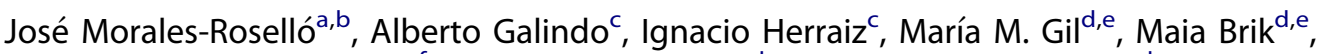 \\ Catalina De Paco-Matallana ${ }^{\mathrm{f}, \mathrm{g}}$, Ricardo Ciammela ${ }^{\mathrm{a}, \mathrm{b}}$, Carlos Sanchez Ajenjo ${ }^{\mathrm{a}, \mathrm{b}}$, \\ Antonio José Cañada Martinez ${ }^{\mathrm{h}}$, Juan Luis Delgado ${ }^{\mathrm{f}, \mathrm{g}}$ and Alfredo Perales-Marín ${ }^{\mathrm{a}, \mathrm{b}}$; \\ (Spanish Group for the Study of Stillbirth)
}

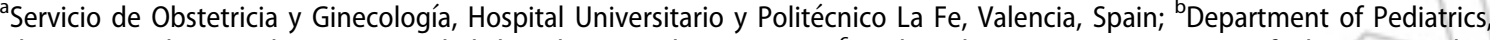
Obstetrics and Gynecology, Universidad de Valencia, Valencia, Spain; ${ }^{C}$ Fetal Medicine Unit, Department of Obstetrics and Gynecology, Maternal and Child Health and Development Network, University Hospital 12 de Octubre, 12 de Octubre Research Institute (imas12). Universidad Complutense de Madrid, Madrid, Spain; ${ }^{\mathrm{d}}$ Department of Obstetrics and Gynecology, Servicio de Obstetricia y Ginecología, Hospital Universitario de Torrejón, Madrid, Spain; 'UFV, Madrid, Spain; fServicio de Obstetricia y Ginecología, Hospital Universitario Virgen de la Arrixaca, Murcia, Spain; ${ }^{9}$ Department of Obstetrics and Gynecology, Universidad de Murcia, Murcia, Spain; hunidad de Bioestadística, Instituto de Investigación Sanitaria La Fe, Valencia, Spain
\end{abstract}

\begin{abstract}
Objective: To examine the potential value of fetal ultrasound and maternal characteristics in the prediction of antepartum stillbirth after 32 weeks' gestation.

Methods: This was a retrospective multicenter study in Spain. In 29 pregnancies, umbilical artery pulsatility index (UA PI), middle cerebral artery pulsatility index (MCA PI), cerebroplacental ratio (CPR), estimated fetal weight (EFW), and maternal characteristics were recorded within 15 days prior to a stillbirth. The values of UA PI, MCA PI, and CPR were converted into multiples of the normal median (MoM) for gestational age and the EFW was expressed as percentile according to a Spanish reference range for gestational age. Data from the 29 pregnancies with stillbirths and 2298 control pregnancies resulting in livebirths were compared and multivariate logistic regression analysis was used to determine significant predictors of stillbirth.

Results: The only significant predictor of stillbirth was CPR (OR $=0.161,95 \%$ confidence interval [Cl] $0.035,0.654 ; p=.014)$; the area under the receiver operating characteristics curve was 0.663 $(95 \% \mathrm{Cl} 0.545,0.782)$ and the detection rate (DR) was $32.14 \%$ at a $10 \%$ false-positive rate (FPR). In addition, when we included MCA and UA PI MoM instead of CPR, only MCA PI MoM was significant $(\mathrm{OR}=0.104,95 \%$ confidence interval $[\mathrm{Cl}] 0.013,0.735 ; p=.029)$, with similar prediction abilities (area under the curve (AUC) 0.645, DR 28.6\%, FPR 10\%).

Conclusions: The CPR and MCA PI are predictors of late stillbirth but the performance of prediction is poor.
\end{abstract}

\section{ARTICLE HISTORY}

Received 26 September 2018 Revised 9 December 2018 Accepted 6 January 2019

\section{KEYWORDS}

Cerebroplacental ratio; fetal growth restriction; fetal hemodynamics; fetal middle cerebral artery Doppler; stillbirth; umbilical artery Doppler

\section{Introduction}

Antepartum stillbirth is reported in 2-4/1000 pregnancies in developed countries and is 10 times higher in the underdeveloped countries [1]. In most stillbirths at $<32$ weeks' gestation, the fetuses are small for gestational age (SGA) and there is evidence of impaired placentation [2,3]. In contrast, in most stillbirths after 32 weeks' gestation, the fetuses are appropriately grown for gestational age (AGA) [4,5]. Some of the AGA stillbirths are associated with abnormalities in fetal Doppler indices, especially in the cerebroplacental ratio (CPR). As the $C P R$ reflexes, the unbalance between fetal needs and placental supply, which characterizes the physiopathology of late-onset fetal growth restriction (FGR), abnormal CPR values would suggest the existence of failure to reach the growth potential (FRGP) [6,7] (fetal growth under optimal conditions). However, despite FRGP, fetuses with abnormal CPR have been proven to be at risk of intrapartum compromise [8-12], the relationship between these hemodynamic disturbances and fetal death has not been yet established.

The objectives of this study is to report fetal Doppler indices obtained within 2 weeks of

CONTACT José Morales-Roselló 0 jose.morales@uv.es $\Theta$ Servicio de Obstetricia@ , Hospital Universitario y Politécnico La Fe, Avenida Fernando Abril Martorell 106, Valencia, Spain 
antepartum stillbirth and develop a model for prediction of such stillbirths from fetal Doppler indices, estimated fetal weight, and maternal characteristics.

\section{Materials and methods}

This was a retrospective multicenter case control study in four university hospitals in Spain. In 29 pregnancies, umbilical artery pulsatility index (UA PI), middle cerebral artery pulsatility index (MCA PI), CPR, estimated fetal weight (EFW), and maternal characteristics were recorded within 15 days prior to antepartum stillbirth. Every stillborn fetus was matched with 80 liveborn fetuses. The 2298 control cases were collected from the same participating hospitals and were randomly selected among the routinely evaluated population at $32-34,35-37,38-40$, and 40-41 weeks' gestation. For each case and control, gestational age was determined from the crown-rump length in the first trimester. Pregnancies complicated by fetal abnormalities or aneuploidies were excluded even when these were found after delivery or in postmortem studies.

The UA PI and MCA PI were evaluated using color Doppler according to standard protocols $[13,14]$ and the CPR was calculated as the ratio between the MCA PI and the UA PI [15].

EFW was obtained from fetal biometry using the Hadlock-4 equation [16]. The values of UA PI, MCA PI, and CPR were converted into multiples of the normal median (MoM) for gestational age by dividing the observed values by the 50th percentile at each gestational age according to the previously published reference ranges: $[14,17]$

$$
\begin{aligned}
& \text { UA PI 50th percentile }=2.2037-0.057955 \times \mathrm{GA} \\
& \quad+0.00053953 \times \mathrm{GA}^{2} \\
& \text { MCA PI 50th percentile }=-3.266164164 \\
& \quad+0.368135209 \times \mathrm{GA}-0.006318278 \times \mathrm{GA}^{2} \\
& \text { CPR 50th percentile }=-3.814786276 \\
& \quad+0.36363249 \times \mathrm{GA}-0.005646672 \times \mathrm{GA}^{2}
\end{aligned}
$$

Where GA is the gestational age expressed in weeks including decimals.

The EFW was expressed as percentile according to a Spanish reference range for gestational age [18].

\section{Statistical analysis}

Descriptive statistics were performed evaluating maternal age, racial origin (Caucasian and nonCaucasian), height and weight (expressed as body mass index, BMI), EFW, birth weight (BW), gravidity (defined as the total number of pregnancies including the current pregnancy and all previous miscarriages), parity (defined as the total number of previous vaginal deliveries and cesarean sections after 24 weeks' gestation), fetal sex, GA at examination, GA at delivery, the interval between examination and delivery, mode of delivery (spontaneous vaginal delivery, instrumental delivery and emergency or elective cesarean section) and Apgar score at $5 \mathrm{~min}$. Median and interquartile range (IQR) were calculated for continuous variables and absolute and relative frequencies were calculated for categorical variables. Comparisons between stillbirths and controls were performed with the chisquare test in the case of categorical variables and the Mann-Whitney $U$ test was carried out in the case of continuous variables.

Multivariate logistic regression analysis was used to determine significant predictors of stillbirth. In these models, the MCA and UA PI MoM were evaluated individually and also in the form of CPR MoM in order to assess the relative importance of each parameter. The Akaike information criterion (AIC) was used to select the best prediction model (the most parsimonious combination) by means of a lower AIC, which indicated the presence of higher accuracy (a difference in the AIC of two units indicated significant differences and a difference of 2-4 units indicated highly significant differences). There is generally a trade-off between goodness of fit and parsimony: low-parsimony models (i.e. models with many parameters) tend to have a better fit than high-parsimony models. This is not usually a good approach, adding more parameters usually results in a good model fit for the data at hand, but that same model will likely be useless for predicting other data sets. The AIC allows a good balance between parsimony and goodness of fit. The results of the logistic regression were reported in the form of odds ratios (OR) with their 95\% confident interval $(\mathrm{Cl})$ and $p$ values. Detection rates (DR) for a false-positive rate (FPR) of 5 and $10 \%$ and ROC analysis with the area under the curve (AUC) were used to evaluate the ability of the model to predict stillbirth. Statistical analysis and graphs were performed using R-Software ${ }^{\circledR} \quad 3.4 .3$ (http://www.r-project.org/). Statistical significance was established at $p<.05$. The authors report no conflict of interests.

\section{Results}

Maternal and pregnancy characteristics in the stillbirths and controls are compared in Table 1. In the stillbirths, compared to livebirths, there was a higher
160

161

162

163

164

165

166

167

168

169

170

171

172

173

174

175

176

177

178

179

180

181

182

183

184

185

186

187

188

189

190

191

192

193

194

195

196

197

198

199

200

201

202

203

204

205

206

207 
Table 1. Comparison between the livebirth and stillbirth groups.

\begin{tabular}{|c|c|c|c|}
\hline Variable & $\begin{array}{l}\text { Livebirth }(N=2298) \\
\text { Median }(I Q R)\end{array}$ & $\begin{array}{l}\text { Stillbirth }(N=29) \\
\text { Median }(\mathrm{IQR})\end{array}$ & $p$-value \\
\hline \multicolumn{4}{|l|}{ Continuous data } \\
\hline BMI $\left(\mathrm{kg} / \mathrm{m}^{2}\right)$ & $23.8(21.5,27.2)$ & $23.5(21.4,25.5)$ & .403 \\
\hline GA at examination (weeks) & $36.43(34.14,38.43)$ & $35.6(34,37.3)$ & .278 \\
\hline EFW (Hadlock-4) (g) & $2724(2266,3149)$ & $2527(2169,2859)$ & .099 \\
\hline EFW centile $^{a}$ & $50(41,61)$ & $44(8,81)$ & .279 \\
\hline Age (years) & $33(29,36)$ & $31(28,36)$ & .374 \\
\hline Parity & $0(0,1)$ & $0(0,1)$ & .942 \\
\hline UA PI MoM & $1.08(0.95,1.24)$ & $1(0.89,1.27)$ & .334 \\
\hline MCA PI MoM & $0.94(0.82,1.09)$ & $0.86(0.68,1.05)$ & .032 \\
\hline CPR MoM & $1.05(0.87,1.26)$ & $0.97(0.68,1.19)$ & .055 \\
\hline GA at delivery (weeks) & $40(39,40.71)$ & $37.14(34.86,38.71)$ & $<.0001$ \\
\hline Interval (days) & $20(7,38)$ & $9(5,10)$ & $<.0001$ \\
\hline$B W(g)$ & $3250(3000,3504)$ & $2700(2180,3240)$ & $<.0001$ \\
\hline BW centile ${ }^{a}$ & $40(20,62)$ & $35(4,74)$ & .955 \\
\hline Apgar 5 min & $10(10,10)$ & $0(0,0)$ & .0001 \\
\hline Variable & $N(\%)$ & $N(\%)$ & $p$-Value \\
\hline \multicolumn{4}{|l|}{ Contingency data } \\
\hline $\mathrm{SGA}\left(\mathrm{BW}<\mathrm{P} 10^{\mathrm{a}}\right)$ & $256(11.14)$ & $8(27.59)$ & .013 \\
\hline CPR MoM $<0.6765^{\mathrm{b}}$ & $148(6.4)$ & $8(27.6)$ & \\
\hline Nulliparity & $1150(50.0)$ & $15(51.7)$ & .857 \\
\hline Apgar 5 min $<7$ & $3(0.13)$ & $29(100)$ & $<.0001$ \\
\hline \multicolumn{4}{|l|}{ Delivery via } \\
\hline Assisted VD. & 464 (20.19) & $2(6.9)$ & .191 \\
\hline Cesarean $\mathrm{S}$. & 487 (21.19) & & \\
\hline Nonassisted VD. & 1347 (58.62) & & \\
\hline \multicolumn{4}{|l|}{ Fetal gender } \\
\hline Female & $1103(48)$ & $12(41.38)$ & .602 \\
\hline Male & $1195(52)$ & $17(58.62)$ & \\
\hline \multicolumn{4}{|l|}{ Ethnicity } \\
\hline Caucasian & $2266(98.61)$ & $25(86.21)$ & $p<.0001$ \\
\hline Noncaucasian & 32 (1.39) & $4(13.8)$ & \\
\hline
\end{tabular}

IQR: interquartile range; BMI: body masss index; SGA: small for gestational age; GA: gestational age; EFW: estimated fetal weight; BW: birth weight; ${ }^{a}$ according to Hospital Clinic de Barcelona references; ${ }^{b}$ CPR MoM values below 0.6765 suggest the presence of failure to reach the growth potential; UA: umbilical artery; MCA: middle cerebral artery; CPR: cerebroplacental ratio; PI; pulsatility index; MoM: multiples of the median; interval: difference between GA at examination and delivery.

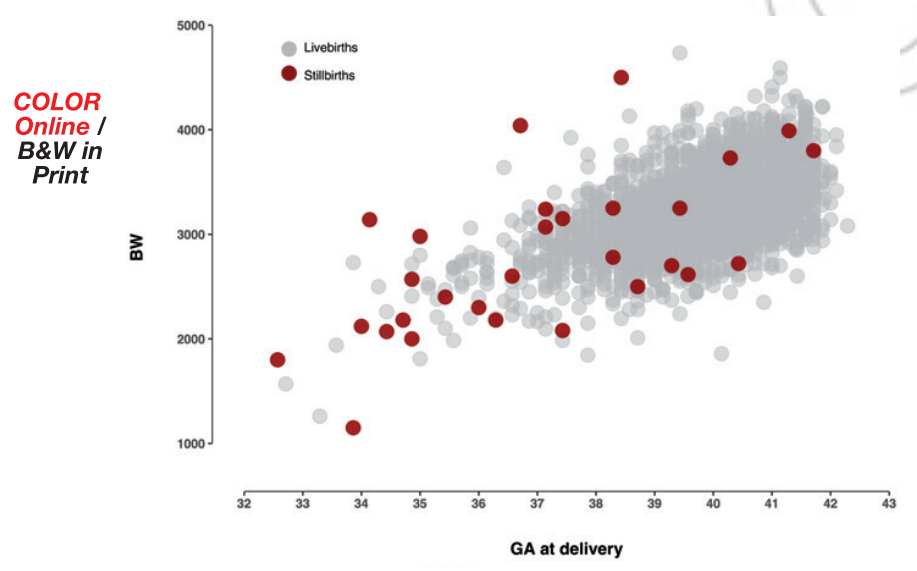

Figure 1. Birthweight in stillbirths (red circles) and livebirths (gray circles) according to gestational age. Stillbirths are situated throughout the entire spectrum of the BW distribution.

incidence of non-Caucasian maternal racial origin, lower MCA PI MoM, lower gestational age at birth, shorter examination to delivery interval, lower median BW and incidence of BW <10th percentile. The BW in the stillbirths and controls are plotted in Figure 1. The BW was $<10$ th percentile in $27.6 \%$ (8 of 29) stillbirths and in $11.1 \%$ (256 of 2298) livebirths.
In the group of stillbirths, 21 (72.4\%) pregnancies had at least one risk factor: $8(27.6 \%)$ were SGA, 2 (6.9\%) had anomalies of the placental insertion (marginal placenta and placenta previa), 5 (17.2\%) had hypertension ( 1 gestational hypertension and 4 preeclampsia), 3 (10.3\%) had diabetes mellitus (1 pregestational and 2 gestational), 4 (13.8\%) had thrombophilia (1 antithrombin III deficit, 1 factor $\mathrm{V}$ Leyden, 1 protein $\mathrm{S}$ deficit, and 1 unspecified) and 1 (3.4\%) had unexplained antepartum hemorrhage from 29 weeks' gestation. However, in 8 cases $(27.6 \%)$, no risk factor was observed, either before or after the baby was born and fetal death was diagnosed during a routine ultrasound examination or after emergency consultation for lack of fetal movements. Regarding the existence of immediate causes of death, detected after consultation, abruption was present in $6(20.7 \%)$ cases. In addition, cord anomalies were seen in three fetuses (10.3\%) (1 velamentous insertion and 2 cord loop compression).

In the multiple regression analysis, the only significant predictor of stillbirth was CPR MoM (Table 2) with an AUC of 0.663 (Figure 2 left). Interestingly, 
Table 2. Model for term stillbirth prediction using CPR.

372

EFW according to local references

\begin{tabular}{lrccccc}
\hline Variables & Estimate & Std. Error & OR & Lower 95\% & Upper 95\% & $p$-value \\
\hline (Intercept) & 2.787 & 3.095 & 16.236 & 0.039 & .03673 .084 & .368 \\
Age & -0.033 & 0.035 & 0.968 & 0.905 & .038 & .348 \\
BMl & -0.022 & 0.046 & 0.979 & 0.888 & .065 & .639 \\
EFW centile & -0.012 & 0.01 & 0.988 & 0.969 & 1.007 & .22 \\
Parity & 0.125 & 0.21 & 1.133 & 0.714 & .63 & .552 \\
Sex & 0.158 & 0.386 & 1.172 & 0.553 & .55 & .681 \\
GA at examination & -0.093 & 0.076 & 0.911 & 0.781 & .056 & .221 \\
CPR MoM & -1.827 & 0.745 & 0.161 & 0.035 & 0.654 & .014
\end{tabular}

Detection rate of $21.43 \%$ for a false-positive rate of $5 \%$

Detection rate of $32.14 \%$ for a false-positive rate of $10 \%$

AUC $0.663,95 \% \mathrm{Cl}[0.545,0.782]$

$\mathrm{AIC}=306.556$

OR: odds ratio; Std. Error: standard error; Lower 95\%: lower limit of the 95\% confidence interval; Upper 95\%: upper limit of the $95 \%$ confidence interval; BMl: body mass index; GA: gestational age; EFW: estimated fetal weight; CPR: cerebroplacental ratio; MoM: multiples of the median; DR: detection rate; FPR: false-positive rate; AUC: area under the ROC curve; $95 \%$ Cl: $95 \%$ confidence interval; AIC: Akaike Information Criterion.
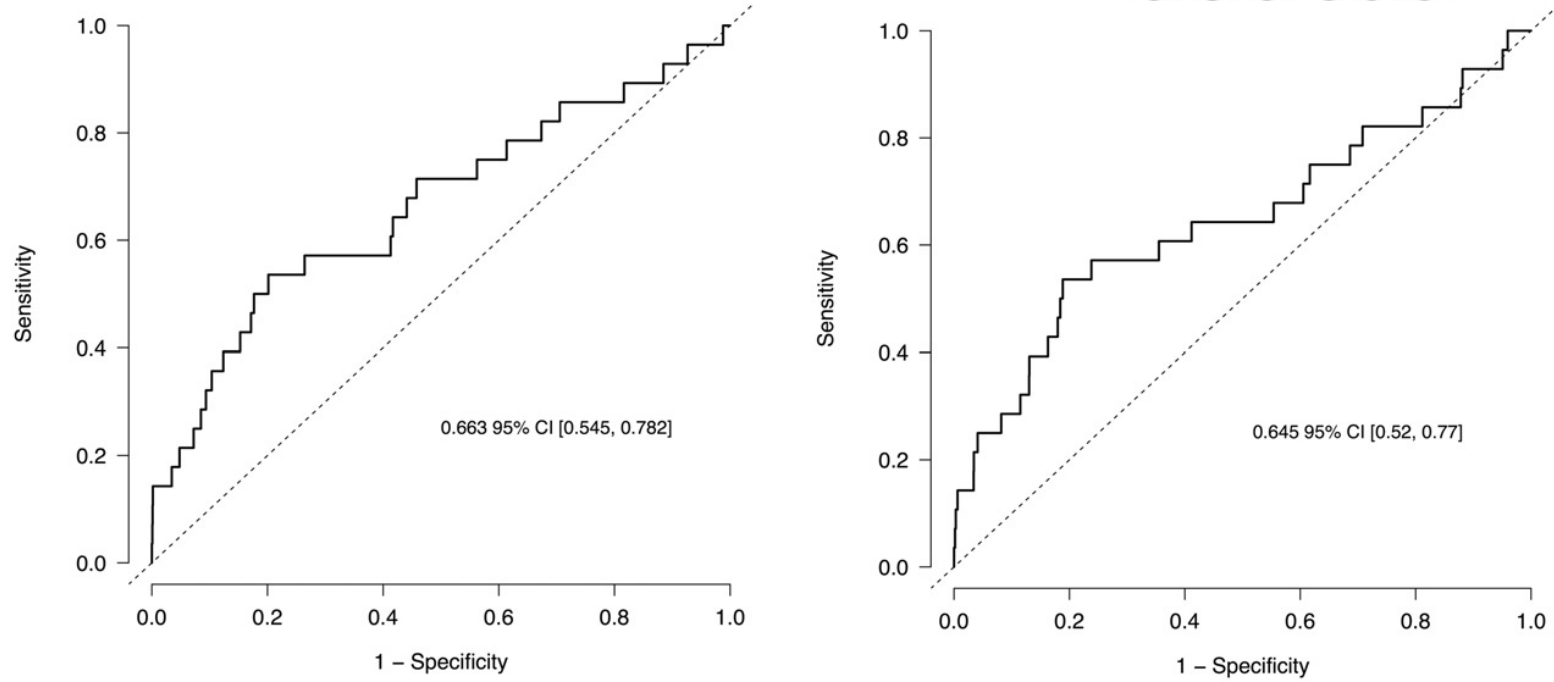

Figure 2. Receiver operating characteristic curves for the prediction of antepartum late stillbirth using multiples of the median for cerebroplacental ratio (left) and middle cerebral artery pulsatility index (right).

$27.6 \%$ of stillborn fetuses presented a CPR below $0.6765 \mathrm{MoM}$ (5th centile of CPR MoM [6]), 6.4\% in the control group, $(p<.0001)$, suggesting the existence of FRGP [6-8] in a considerable proportion of the cases (Figure 3). In order to better evaluate the importance of CPR MoM, we studied its components (UA PI and MCA PI MoM) separately in a second multivariate analysis (Table 3); in this model, only MCA MoM provided significant prediction of stillbirth and the AUC for prediction of stillbirth was 0.645 (Figure 2 right).

\section{Discussion}

\section{Main findings of the study}

This multicenter study of late antepartum stillbirths with antenatal assessment within 2 weeks of the adverse event has demonstrated that more than $70 \%$

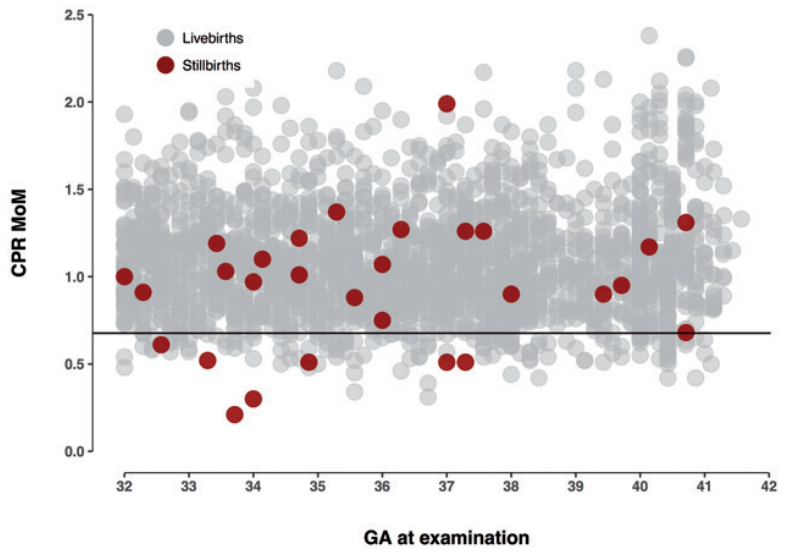

Figure 3. Scatter plot representing the CPR MoM according to gestational age. A notable proportion of stillborn fetuses (27.6\%) present values below 0.6765 ( $<5$ th centile) suggesting the existence of a failure to reach the growth potential $(6.4 \%$ in the control group, $p<.0001$ ). 
Table 3. Model for stillbirth prediction using MCA PI and UA PI.

\begin{tabular}{|c|c|c|c|c|c|c|}
\hline \multicolumn{7}{|c|}{ EFW according to local references } \\
\hline Variables & Estimate & Std. Error & OR & Lower $95 \%$ & Upper 95\% & $p$-value \\
\hline (Intercept) & 1.09 & 3.212 & 2.975 & 0.006 & 1759.513 & .734 \\
\hline Age & -0.033 & 0.035 & 0.968 & 0.904 & 1.038 & .348 \\
\hline BMl & -0.023 & 0.046 & 0.977 & 0.887 & 1.064 & .623 \\
\hline EFW centile & -0.012 & 0.01 & 0.988 & 0.969 & 1.008 & .234 \\
\hline Parity & 0.117 & 0.211 & 1.124 & 0.707 & 1.62 & .578 \\
\hline Sex & 0.123 & 0.387 & 1.131 & 0.532 & 2.465 & .750 \\
\hline GA at examination & -0.066 & 0.078 & 0.937 & 0.801 & 1.09 & .401 \\
\hline UA PI MoM & 0.864 & 0.657 & 2.372 & 0.563 & 8.235 & .189 \\
\hline MCA PI MoM & -2.26 & 1.038 & 0.104 & 0.013 & 0.735 & .029 \\
\hline \multicolumn{7}{|c|}{ Detection rate of $25 \%$ for a false-positive rate of $5 \%$} \\
\hline \multicolumn{7}{|c|}{ Detection rate of $28.6 \%$ for a false-positive rate of $10 \%$} \\
\hline \multicolumn{7}{|c|}{ AUC $0.645,95 \% \mathrm{Cl}[0.52,0.77]$} \\
\hline \multicolumn{7}{|c|}{$\mathrm{AIC}=308.49$} \\
\hline
\end{tabular}

OR: odds ratio; Std. Error: standard error; Lower 95\%: lower limit of the 95\% confidence interval; Upper 95\%: upper limit of the $95 \%$ confidence interval; BMI: body mass index; GA: gestational age; EFW: estimated fetal weight; CPR: cerebroplacental ratio; MoM: multiples of the median; DR: detection rate; FPR: false-positive rate; AUC: area under the ROC curve; $95 \%$ Cl: 95\% confidence interval; AIC: Akaike Information Criterion.

are not SGA and that the only antenatal predictor of stillbirth is MCA PI. The performance of screening by MCA PI or CPR for the prediction of stillbirth is poor with DR of about $30 \%$ at FPR of $10 \%$.

\section{Interpretation of the findings and review of earlier studies}

Previous studies reported that the risk of stillbirth increases with maternal age and BMI and it is also increased in primigravidas and multiparas [19-33]. Our results demonstrate that once CPR or MCA PI are taken into account, these maternal characteristics have no significant influence on stillbirth. Similarly, previous studies suggested that stillbirth is more common in male than female fetuses [34-37], but we found that fetal gender did not have a significant contribution to stillbirth. Finally, fetal smallness has also been related with stillbirth in earlier studies $[19,33]$. In this work, we did not find EFW to be relevant. In fact, according to recent publications, the true influence of smallness on stillbirth might be even lower than previously thought, as it could be due to dehydration processes leading to a quick loss of weight prior to delivery [38].

Our findings on the association between low MCA PI and CPR with stillbirth are in agreement with the results of previous studies [6-13,39,40], which relates adverse outcome with the existence of FRGP [6-8]. Conversely, other studies have not found CPR to be an important predictor of stillbirth $[41,42]$. The explanation for this controversy may be due to the type of study population; CPR may be very useful in pregnancies at high risk of FGR [39], but less in low-risk populations $[5,41]$. Further studies are required to clarify these associations.

\section{Clinical and research implications}

Assessment of fetal hemodynamics provides poor prediction of stillbirth. However, pregnancies with lowfetal MCA PI and CPR may require close followup because in some cases, there is increased risk of stillbirth. Further research is necessary to identify new potential markers that may improve prediction of late stillbirth.

\section{Study strengths and limitations}

The major strength of the study is the recording of data on fetal hemodynamics within a short time interval before fetal death. The limitations of the study are the low number of cases and the retrospective nature of the study, which avoided retrieval of some data in all cases, including maternal smoking, which is reported to be an important contributor to stillbirth [19].

In conclusion, MCA PI and CPR are the relevant parameters in the explanation of late antepartum fetal death. However, due to the weakness of the associations, the ability of the models to predict stillbirth remains poor.

\section{Disclosure statement}

No potential conflict of interest was reported by the authors.

\section{References}

[1] Cousens S, Blencowe $H$, Stanton $C$, et al. National, regional, and worldwide estimates of stillbirth rates in 2009 with trends since 1995: a systematic analysis. Lancet. 2011;377:1319-1330. 
[2] Poon LC, Tan MY, Yerlikaya G, et al. Birth weight in live births and stillbirths. Ultrasound Obstet Gynecol. 2016;48:602-606.

[3] Zhang X, Joseph KS, Cnattingius S, et al. Birth weight differences between preterm stillbirths and live births: analysis of population-based studies from the U.S. and Sweden. BMC Pregnancy Childbirth. 2012;12:119.

[4] Poon LC, Volpe N, Muto B, et al. Birthweight with gestation and maternal characteristics in live births and stillbirths. Fetal Diagn Ther. 2012;32:156-165.

[5] Bakalis S, Akolekar R, Gallo DM, et al. Umbilical and fetal middle cerebral artery Doppler at 30-34 weeks' gestation in the prediction of adverse perinatal outcome. Ultrasound Obstet Gynecol. 2015;45:409-420.

[6] Morales-Roselló J, Khalil A, Morlando $M$, et al. Changes in fetal Doppler indices as a marker of failure to reach growth potential at term. Ultrasound Obstet Gynecol. 2014;43:303-310.

[7] Morales-Roselló J, Khalil A. Fetal cerebral redistribution: a marker of compromise regardless of fetal size. Ultrasound Obstet Gynecol. 2015;46:385-388.

[8] Morales-Roselló J, Khalil A, Morlando M, et al. Poor neonatal acid-base status in term fetuses with low cerebroplacental ratio. Ultrasound Obstet Gynecol. 2015;45:156-161.

[9] Khalil AA, Morales-Rosello J, Morlando M, et al. Is fetal cerebroplacental ratio an independent predictor of intrapartum fetal compromise and neonatal unit admission? Am J Obstet Gynecol. 2015;213: 54.e1-54.e10.

[10] Khalil AA, Morales-Rosello J, Elsaddig $M$, et al. The association between fetal Doppler and admission to neonatal unit at term. Am J Obstet Gynecol. 2015; 213:57.e1-57.e7.

[11] Prior T, Paramasivam G, Bennett $P$, et al. Are fetuses that fail to achieve their growth potential at increased risk of intrapartum compromise? Ultrasound Obstet Gynecol. 2015;46:460-464.

[12] Prior T, Mullins E, Bennett $P$, et al. Prediction of intrapartum fetal compromise using the cerebroumbilical ratio: a prospective observational study. Am J Obstet Gynecol. 2013;208:124.e1-124.e6.

[13] Morales Roselló J, Hervás Marín D, Perales Marín A, et al. Doppler study of the fetal vertebral and middle cerebral arteries in fetuses with normal and increased umbilical artery resistance indices. J Clin Ultrasound. 2013;41:224-229.

[14] Acharya G, Wilsgaard T, Berntsen GK, et al. Reference ranges for serial measurements of umbilical artery Doppler indices in the second half of pregnancy. Am J Obstet Gynecol. 2005;192:937-944.

[15] Baschat AA, Gembruch U. The cerebroplacental Doppler ratio revisited. Ultrasound Obstet Gynecol. 2003;21:124-127.

[16] Hadlock FP, Harrist RB, Carpenter RJ, et al. Sonographic estimation of fetal weight. The value of femur length in addition to head and abdomen measurements. Radiology. 1984;150:535-540.

[17] Morales-Roselló J, Khalil A, Morlando M, et al. Doppler reference values of the fetal vertebral and middle cerebral arteries, at 19-41 weeks gestation. J Matern Fetal Neonatal Med. 2015;28:338-343.
[18] Figueras F, Meler E, Iraola A, et al. Customized birthweight standards for a Spanish population. Eur J Obstet Gynecol Reprod Biol. 2008;136:20-24.

[19] Flenady V, Koopmans L, Middleton P, et al. Major risk factors for stillbirth in high-income countries: a systematic review and meta-analysis. Lancet. 2011;377: 1331-1340.

[20] Man J, Hutchinson JC, Ashworth M, et al. Stillbirth and intrauterine fetal death: contemporary demographic features of $>1000$ cases from an urban population. Ultrasound Obstet Gynecol. 2016;48:591-595.

[21] Nohr EA, Bech BH, Davies MJ, et al. Prepregnancy obesity and fetal death: a study within the Danish National Birth Cohort. Obstet Gynecol. 2005;106: 250-259.

[22] Felisbino-Mendes MS, Matozinhos FP, Miranda JJ, et al. Maternal obesity and fetal deaths: results from the Brazilian cross-sectional demographic health Survey, 2006. BMC Pregnancy Childbirth 2014;14:5.

[23] Chu SY, Kim SY, Lau J, et al. Maternal obesity and risk of stillbirth: a metaanalysis. Am J Obstet Gynecol. 2007;197:223-228.

[24] Kristensen J, Vestergaard $M$, Wisborg $K$, et al. Prepregnancy weight and the risk of stillbirth and neonatal death. BJOG. 2005;112:403-408.

[25] Aune D, Saugstad OD, Henriksen T, et al. Maternal body mass index and the risk of fetal death, stillbirth, and infant death: a systematic review and meta-analysis. JAMA. 2014;311:1536-1546.

[26] Gordon A, Raynes-Greenow C, McGeechan K, et al. Risk factors for antepartum stillbirth and the influence of maternal age in New South Wales Australia: a population based study. BMC Pregnancy Childbirth 2013;13:12.

[27] Lean SC, Derricott $H$, Jones RL, et al. Advanced maternal age and adverse pregnancy outcomes: a systematic review and meta-analysis. Plos One. 2017;12: e0186287.

[28] Waldenström U, Cnattingius S, Norman $M$, et al. Advanced maternal age and stillbirth risk in nulliparous and parous women. Obstet Gynecol. 2015;126: 355-362.

[29] Bukowski R, Carpenter M, Conway D, et al. Stillbirth Collaborative Research Network Writing Group. Association between stillbirth and risk factors known at pregnancy confirmation. JAMA. 2011;306: 2469-2479.

[30] Khalil A, Syngelaki A, Maiz N, et al. Maternal age and adverse pregnancy outcome: a cohort study. Ultrasound Obstet Gynecol. 2013;42:634-643.

[31] Marozio L, Picardo E, Filippini C, et al. Maternal age over 40 years and pregnancy outcome: a hospitalbased survey. J Matern Fetal Neonatal Med. 2017:1-7.

[32] Bai J, Wong FW, Bauman A, et al. Parity and pregnancy outcomes. Am J Obstet Gynecol. 2002;186: 274-278.

[33] Gardosi J, Madurasinghe V, Williams M, et al. Maternal and fetal risk factors for stillbirth: population based study. BMJ 2013;346:f108.

[34] Mondal D, Galloway TS, Bailey TC, et al. Elevated risk of stillbirth in males: systematic review and meta- 
analysis of more than 30 million births. BMC Med. 2014;12:220.

[35] Di Renzo GC, Rosati A, Sarti RD, et al. Does fetal sex affect pregnancy outcome? Gend Med. 2007;4:19-30.

[36] Antonakou A, Papoutsis D. The effect of fetal gender on the delivery outcome in primigravidae women with induced labours for all indications. J Clin Diagn Res. 2016;10:QC22-QC25.

[37] Naeye RL, Burt LS, Wright DL, et al. Neonatal mortality, the male disadvantage. Pediatrics. 1971;48: 902-906.

[38] Man J, Hutchinson JC, Ashworth M, et al. Effects of intrauterine retention and postmortem interval on body weight following intrauterine death: implications for assessment of fetal growth restriction at autopsy. Ultrasound Obstet Gynecol. 2016;48:574-578.
[39] Khalil A, Morales-Roselló J, Townsend R, et al. Value of third-trimester cerebroplacental ratio and uterine artery Doppler indices as predictors of stillbirth and perinatal loss. Ultrasound Obstet Gynecol. 2016;47: 74-80.

[40] Morales-Roselló J, Khalil A, Akhoundova F, et al. Fetal cerebral and umbilical Doppler in pregnancies complicated by late-onset placental abruption. J Matern Fetal Neonatal Med. 2017;30:1320-1324.

[41] Monaghan C, Binder J, Thilaganathan B, et al. Perinatal loss at term: role of uteroplacental and fetal Doppler assessment. Ultrasound Obstet Gynecol. 2018;52:72-77.

[42] Akolekar R, Syngelaki A, Gallo DM, et al. Umbilical and fetal middle cerebral artery Doppler at 35-37 weeks. Ultrasound Obstet Gynecol. 2015;46:82-92.
690

691

692

693

694

695

696

697

698

699

700

701

702

703

704

705

706

707

708

709

710

711

712

713

714

715

716

717

718

719

720

721

722

723

724

725

726

727

728

729

730

731

732

733

734

735

736

737

738

739

740

741

742 